\title{
Classical Gravity on Fuzzy Space-Time
}

\author{
J. Madore \\ Laboratoire de Physique Théorique et Hautes Energies* \\ Université de Paris-Sud, Bât. 211, F-91405 Orsay
}

December 6, 2017

\begin{abstract}
A review is made of recent efforts to find relations between the commutation relations which define a noncommutative geometry and the gravitational field which remains as a shadow in the commutative limit.
\end{abstract}

\section{Historical Introduction}

The position $x$ and the momentum $p$ of a classical particle can be simultaneously measured and $(x, p)$ defines a point in classical phase-space. The set of polynomials in the variables $(x, p)$ can be added and multiplied; they form a commutative algebra. When the particle is quantized the points disappear; because of the Heisenberg uncertainty relations the two operators $\hat{x}$ and $\hat{p}$ can no longer be simultaneously measured. However, it still makes sense to consider the algebra of polynomials in them. It is a noncommutative algebra which Dirac [1] referred to as the 'quantum algebra'.

A vector in classical phase space can be naturally identified with a derivation of the algebra of polynomials and as such can be generalized to the quantized case. Dirac called it a 'quantum differentiation'. From the correspondence principle we see that

$$
\lim _{\hbar \rightarrow 0} \frac{i}{\hbar}[\hat{p}, \hat{f}] \rightarrow \frac{\partial f}{\partial x}, \quad \lim _{\hbar \rightarrow 0} \frac{i}{\hbar}[\hat{x}, \hat{f}] \rightarrow-\frac{\partial f}{\partial p} .
$$

The $\hat{f}$ is here an arbitrary element of the 'quantum algebra'. The quantized version of the vector $X=\left(X_{x}, X_{p}\right)$ is then the derivation of the algebra given by

$$
\hat{X} f=\frac{i}{\hbar} \hat{X}_{x}[\hat{p}, \hat{f}]-\frac{i}{\hbar} \hat{X}_{p}[\hat{x}, \hat{f}]
$$

Because of the ordering problem we see that a 'quantum derivation' defines a unique vector but that the inverse is not true.

Some decades later von Neumann introduced the term 'noncommutative geometry' to refer in general to a geometry in which an algebra of functions is replaced by a noncommutative algebra. As in the phase-space example coordinates are replaced by generators of the algebra. Since these do not commute they cannot be simultaneously diagonalized and

*Laboratoire associé au CNRS, URA D0063 
the space disappears. We shall argue with an example below that, just as Bohr cells replace classical-phase-space points, the appropriate intuitive notion to replace a 'point' is a 'cell'.

But 'geometry' is more than a set of points and so more is needed that just an algebra. This problem was solved by Connes [2, 3] who introduced the notion of 'noncommutative differential geometry' to refer in general to a noncommutative geometry with an associated differential calculus. Just as it is possible to give many differential structures to a given topological space it is possible to define many differential calculi over a given algebra. We shall use the term 'noncommutative geometry' to mean 'noncommutative differential geometry' in the sense of Connes.

In order to cope with the divergences in the newly-discovered quantum field theory, throughout the 1930's Heisenberg and others flirted with the idea of replacing space-time with a fundamental lattice. The lattice spacing would serve as cut-off. The difficulty here is that the lattice destroys Lorentz invariance. A solution to this problem was proposed some time later by Snyder [四] who showed that by using noncommutative 'coordinates' it was possible to have a version of 'space-time' which had some of the desirable features of a lattice but which was nevertheless Lorentz invariant.

When referring to the version of space-time which we shall describe here we use the adjective 'fuzzy' to underline the fact that points are ill-defined [5, 6]. Since the algebraic structure is described by commutation relations the qualifier 'quantum' was used by Snyder and by others [7, 8]. This latter expression is unfortunate since the structure has no immediate relation to quantum mechanics and also it leads to confusion with a 'space-time' on which a 'quantum' Lorentz group acts [9].

It is worth mentioning something which we do not mean by the expression 'fuzzy spacetime'. To explain the Zitterbewegung of an electron Schrödinger and others considered center-of-mass position operators of the form

$$
q^{i}=\hat{x}^{i}+m^{-2}(\hat{S} \times \hat{p})^{i} .
$$

Here $\hat{S}$ is the spin polarization vector. Because of the canonical commutation relations the operators $q^{i}$ do not commute. One could introduce an algebra generated by them, for each value of the time coordinate, and even a differential calculus to construct a noncommutative geometry. The algebra of the commutative limit would be however simply the real (or complex) numbers, the possible values of a function at the position of the particle; it would not be an algebra of functions on space-time. An associated noncommutative geometry would be a noncommutative generalization of a point. This example has a membrane generalization. The fact that the target-space coordinates of an extended object do not commute does not mean that the noncommutative algebra which they generate is a noncommutative version of space-time. It could however be considered as a noncommutative Kaluza-Klein extension of a point of ordinary space-time. For example if one truncates [10] the modes of a membrane and modifies the product to construct a matrix algebra the resulting geometry [11] is a matrix extension of space-time. Conversely a static 'membrane' in a fuzzy space-time as we define it can only have a finite number of modes and will be described by some finite-dimensional quotient algebra. 


\section{Fuzzy Space-Time}

By a 'fuzzy' space-time we mean an algebra with 4 generators $q^{\mu}$ which do not commute:

$$
\left[q^{\mu}, q^{\nu}\right]=i \mu_{P}^{-2} q^{\mu \nu}
$$

In the limit where the Planck mass $\mu_{P}$ tends to infinity we wish to identify the generators with the coordinates of ordinary Minkowski space-time:

$$
\lim _{\mu_{P} \rightarrow \infty} q^{\mu}=x^{\mu}
$$

The structure of the algebra is given, for example, by the commutation relations $\left[q^{\lambda}, q^{\mu \nu}\right]$. Several possibilities have been considered [4, 6, 7].

There are several reasons for which one might be lead to consider fuzzy space-times. Effectively when one computes for example Feynman diagrams one is forced to introduce a cut-off $\Lambda$, which can certainly be taken to be less than the Planck mass. This introduces a fuzziness in the definition of space-time points of the order of at least $\mu_{P}^{-1}$. Since this is an unavoidable feature of particle physics one might as well include it as part of the geometry of space-time. It can be argued [12] that noncommuting coordinates are a natural consequence of the quantum fluctuations of the gravitational field. We would like to argue in fact that the commutation relations of the algebra determine and are determined by the gravitational field. The gravitational field can be then considered to regularize the ultraviolet divergences through the introduction of a noncommutative structure on space-time. We have already mentioned the motivation of Snyder to introduce a Lorentz-invariant version of a lattice. The most compelling argument however in favour of studying fuzzy versions of space-time is that they constitute a natural generalization of ordinary space-time and they can be studied. It is possible to generalize to the noncommutative case the notions of vectors, covectors, metrics, connections etc.

\subsection{Problems}

A satisfactory definition of the noncommutative extension of Riemannian curvature has not yet been found [13, 14]. Invariants are another problem. Cyclic cohomology has been used [2, 3] to define topological invariants but the ordinary invariants which might lead to an action principle have not been found. Indeed we are not in a position to argue that there is even a valid action principle. A discussion of this point has been made by Connes and co-workers in a series of articles 15, 16, 17, 18 but the definition which these authors propose is valid only on the noncommutative generalizations of compact spaces with euclidean-signature metrics.

\subsection{General relations}

Consider the algebra generated by the 'coordinates' $q^{\mu}$ and the following diagram

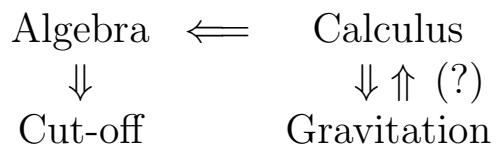

The top arrow is a mathematical triviality. We shall define it in the next section when we recall the definition of a differential calculus. The left arrow is the 'pointlessness' property of 
noncommutative geometry. Since points have been eliminated there can be no divergences [4, 6, 7]. As mentioned above this is one of the principal motivations of studying noncommutative geometry. The relations expressed by the right arrow are new results [19, 8]. We shall argue below using simple models that the gravitational field is determined by the differential calculus. We would like to argue that the inverse is also true but this is less certain. If we could argue that there is a 'natural' differential calculus over every 'natural' noncommutative generalization of space-time then we could conclude that the commutation relations of the algebra determine and are determined by the gravitational field. In any case to within the uncertainty expressed by the question mark the diagram expresses a possible realization of the old idea of Pauli [20, 21] that the gravitational field can be used to regularize ultraviolet divergences.

\section{Differential Calculi}

We recall that a differential calculus over an algebra $\mathcal{A}$ is another (graded) algebra

$$
\Omega^{*}(\mathcal{A})=\bigcup_{p} \Omega^{p}(\mathcal{A})
$$

which gives a differential structure to $\mathcal{A}$. The elements of $\Omega^{p}(\mathcal{A})$ are called $p$-forms. There is a linear map $d$ which takes $p$-forms into $(p+1)$-forms and which satisfies a graded Leibniz rule as well as the condition $d^{2}=0$. By definition $\Omega^{0}(\mathcal{A})=\mathcal{A}$. In general there can be several differential calculi over a given algebra. For a more detailed discussion within the context of noncommutative geometry we refer to the primary sources [2, 3] or to secondary sources, for example, [6, 22].

In ordinary geometry the 1 -forms $\Omega^{1}(\mathcal{A})$ are dual to the vector fields. There is also an interesting relation between the differential $d$ and the Dirac operator $\not D$ which we mention. Let $\psi$ be a Dirac spinor and $f$ a smooth function. It is straightforward to see that

$$
\partial_{\lambda} f \gamma^{\lambda} \psi=-[\not \not D, f] \psi
$$

If we make the replacement $\gamma^{\lambda} \mapsto d x^{\lambda}$ the left-hand side becomes equal to $d f \psi$ and we can write the differential as a commutator:

$$
d f=-[\not \not D, f] .
$$

It would be natural to try to generalize this relation to higher-order forms by using a graded commutator on the right-hand side. Because $d x^{\mu} d x^{\nu}+d x^{\nu} d x^{\mu}=0$ whereas $\gamma^{\mu} \gamma^{\nu}+\gamma^{\nu} \gamma^{\mu} \neq 0$ one would find that $d^{2} \neq 0$. We shall encounder a similar problem in a model in the next section.

\section{Some Simple Modles}

The simplest examples of noncommutative algebras are furnished by matrix algebras. A less trivial example is furnished by the quantum plane. 


\subsection{The Connes-Lott models}

Consider the algebra $M_{2}(\mathbb{C})$ of $2 \times 2$ complex matrices which we write as $M_{2}(\mathbb{C})=M_{2}^{+} \oplus M_{2}^{-}$ where $M_{2}^{+}$is the algebra of diagonal matrices and $M_{2}^{-}$is the set of off-diagonal matrices. Set $\mathcal{A}=M_{2}^{+}$and define [23] a differential calculus over $\mathcal{A}$ by

$$
\Omega^{2 p}(\mathcal{A})=M_{2}^{+}, \quad \Omega^{2 p+1}(\mathcal{A})=M_{2}^{-}
$$

for all $p \geq 0$. Let $\eta$ be any anti-hermitian element of $M_{2}^{-}$. One can define a differential by

$$
d \alpha=-[\eta, \alpha]
$$

where the commutator is a graded commutator. The $\eta$ is a generalized Dirac operator. If one chooses $\eta^{2}=-1$ it follows that $d^{2}=0$. One can think of $\mathcal{A}$ as the algebra of functions on 2 points and the differential as the finite-difference operator.

A slightly less trivial example is furnished by the algebra $M_{3}(\mathbb{C})$ of $3 \times 3$ complex matrices which we write as $M_{3}(\mathbb{C})=M_{3}^{+} \oplus M_{3}^{-}$where $M_{3}^{+}=M_{2}(\mathbb{C}) \times \mathbb{C}$ is the algebra of blockdiagonal matrices and $M_{3}^{-}$is the remainder. Set $\mathcal{A}=M_{3}^{+}$and define [23] a differential calculus over $\mathcal{A}$ by

$$
\Omega^{0}(\mathcal{A})=M_{3}^{+}, \quad \Omega^{1}(\mathcal{A})=M_{3}^{-}, \quad \Omega^{2}(\mathcal{A})=\mathbb{C}
$$

and $\Omega^{p}(\mathcal{A})=0, p \geq 3$. Let $\eta$ be any anti-hermitian element of $M_{3}^{-}$. One can define again a differential as above. It is not possible however to impose the condition $\eta^{2}=-1$ and it was necessary to define the 2 -forms as the projection of $M_{3}^{+}$onto the single factor $\mathbb{C}$ in order to have the relation $d^{2}=0$. The problem here is similar to that in the ordinary case where also $\not^{2} \neq 1$. One can think of $\mathcal{A}$ again as an algebra of functions on two points but with an additional algebraic structure on one of the points. The differential can no longer be considered as a finite-difference operator.

\subsection{Derivation-based models}

Consider $\mathcal{A}=M_{2}(\mathbb{C})$ and let $\Omega^{*}(\mathcal{A})$ be the differential calculus [24, 11] based on the Lie algebra of all derivations of $\mathcal{A}$. In this case $\Omega^{1}(\mathcal{A})$ has an anti-commuting basis of 3 elements $\theta^{i}$ which commute with the elements of the algebra:

$$
f \theta^{i}=\theta^{i} f, \quad \theta^{i} \theta^{j}=-\theta^{j} \theta^{i} .
$$

An arbitrary element $\xi$ of $\Omega^{1}(\mathcal{A})$ can be written in the form $\xi=\xi_{i} \theta^{i}$ where the $\xi_{i}$ are elements of $\mathcal{A}$. Let $\lambda_{i}$ be the (suitably normalized) Pauli matrices. The special 1 -form $\theta=-\lambda_{i} \theta^{i}$ is a generalized Dirac operator. The first Connes-Lott model is a singular contraction of this one [25].

The algebra $M_{2}(\mathbb{C})$ can be used to furnish a very simple example of what we meant in the Introduction by an 'invariant version of a lattice'. Consider the ordinary round 2sphere in 3 dimensions and as an extreme lattice approximation consider the north and south poles. Functions on these two points can be put in one-to-one correspondence with diagonal $2 \times 2$ matrices. The lattice approximation has as algebra of functions a (commutative) 2-dimensional algebra.

There is however a more interesting approximation which consists in considering the 2sphere as a classical spin and approximating it by a spin $1 / 2$. The corresponding algebra of observables is the algebra of all $2 \times 2$ matrices. In general two observables cannot be 
simultaneously measured but any single observable has 2 eigenvalues which correspond to its value on the two 'points'. The lattice algebra is obviously not invariant under the adjoint action of the rotation group but the full matrix algebra is invariant.

The algebra $\mathcal{A}=M_{n}(\mathbb{C})$ of $n \times n$ complex matrices can be used to construct an $\mathrm{SO}_{3}$ invariant 'lattice' approximation to the sphere $S^{2}$ with 'lattice spacing' proportional to $1 / n$. There are no points but the sphere is divided into $n$ cells. This 'fuzzy sphere' has been studied from several points of view [5, 6, 26, 27, 28, 29] and a super-symmetric version has been proposed [30].

\subsection{The quantum plane}

The quantum plane is the algebra $\mathcal{A}$ generated by 'variables' $x$ and $y$ which satisfy the relation

$$
x y=q y x
$$

where $q$ is an arbitrary complex number. It has over it a (Wess-Zumino) calculus $\Omega^{*}(\mathcal{A})$ 31, 32 which is invariant under the action of a quantum group. An arbitrary element $\xi$ of $\Omega^{1}(\mathcal{A})$ can be written uniquely in the form $\xi=\xi_{x} d x+\xi_{y} d y$ where $\xi_{x}$ and $\xi_{y}$ are elements of $\mathcal{A}$ but the basis elements $d x$ and $d y$ do not anti-commute and they do not commute with the elements of the algebra. There is no generalized Dirac operator.

\subsection{The extended quantum plane}

One can extend the previous algebra by adding the inverses $x^{-1}$ and $y^{-1}$. For each integer $n$ and each set of $n$ linear-independent elements $\lambda_{i}$ of $\mathcal{A}$, there exists then a differential calculus $\Omega^{*}(\mathcal{A})$ [19, 8 based on the derivations

$$
e_{i} f=\left[\lambda_{i}, f\right]
$$

The differential of an element of $f$ of $\mathcal{A}$ is defined as in the usual case by the identity

$$
d f\left(e_{i}\right)=e_{i} f .
$$

The $\Omega^{1}(\mathcal{A})$ has a preferred basis of $n$ elements $\theta^{i}$ which commute with the elements of the algebra. The relations of the calculus can be written in the form

$$
\theta^{i} \theta^{j}=P^{i j}{ }_{k l} \theta^{k} \theta^{l}
$$

where the $P^{i j}{ }_{k l}$ are functions of $q$. In this case also the special 1 -form $\theta=-\lambda_{i} \theta^{i}$ is a generalized Dirac operator. If one accepts as definition of 'dimension' the rank of $\Omega^{1}(\mathcal{A})$ then for $q \neq 1$ the 'dimension' of the extended quantum plane can be arbitrary. Unless however $n=2$ the differential calculus has a singular limit as $q \rightarrow 1$. For $n=2$ and a special choice of the elements $\lambda_{i}$ the resulting differential calculus is an extension of the Wess-Zumino calculus. The $\theta$ cannot however be considered as an element of the Wess-Zumino calculus since the $\theta^{i}$ are constructed using the inverses of $x$ and $y$.

\subsection{Quantum groups}

Consider the quantum groups $G L_{q}(n)$ with generators $T_{j}^{i}$ and antipode $\kappa$. The left invariant 1-forms

$$
\omega_{j}^{i}=\kappa\left(T_{k}^{i}\right) d T_{j}^{k}
$$

generate 33 a bicovariant differential calculus $\Omega^{*}\left(G L_{q}(n)\right)$. There is a right- and leftinvariant generalized Dirac operator. 


\section{Kaluza-Klein Theory}

One can consider a modified version of Kaluza-Klein with a noncommutative algebra to describe the extra hidden 'dimensions' and study electromagnetism or gravity on the extended structure. There have been numerous models based on this idea. With electromagnetism one can obtain probably any Yang-Mills-Higgs-Kibble model by appropriately chosing the noncommutative factor of the differential calculus. The Higgs potential appears as the term of the electromagnetic action associated with the hidden 'directions'. The length scale of the extra texture which space-time aquires must be of the order of the weak-boson Compton wave length.

With gravity the situation is more rigid. No matter what the algebra and the differential calculus, Yang-Mills potentials can be only associated with those 'directions' in the hidden 'dimensions' for which the 1-forms commute with all the elements of the algebra. The length scale must be here of the order of the Planck length. We refer, for example, to a recent review article [22] for further details and references to the original literature.

\section{Classical Gravity}

Classical gravity can be introduced in different ways. We shall define it in a way which seems best suited to a noncommutative generalization. The algebra $\mathcal{A}$ is the algebra of smooth functions on the space-time manifold. As principal steps we mention the following possible list:

1. Introduce a moving frame (Vierbein) $\theta^{\alpha}$ which is a basis of $\Omega^{1}(\mathcal{A})$. We here suppose for simplicity that the manifold is parallelizable.

2. Define a metric using the frame

$$
g\left(\theta^{\alpha} \otimes \theta^{\beta}\right)=g^{\alpha \beta}
$$

where the $g^{\alpha \beta}$ are given elements of $\mathcal{A}$.

3. Define a covariant derivative on the frame

$$
D \theta^{\alpha}=-\omega^{\alpha}{ }_{\beta \gamma} \theta^{\beta} \otimes \theta^{\gamma}
$$

where the $\omega^{\alpha}{ }_{\beta \gamma}$ are given elements of $\mathcal{A}$ and extend to arbitrary 1 -forms $\xi=\xi_{\alpha} \theta^{\alpha}$ using the Leibniz rule

$$
D\left(\xi_{\alpha} \theta^{\alpha}\right)=d \xi_{\alpha} \otimes \theta^{\alpha}+\xi_{\alpha} D \theta^{\alpha}
$$

4. Require that the torsion vanish.

5. Require that the connection be metric.

6. Define the curvature in terms of the second covariant derivative $D^{2}$ and contract indices to form the Ricci scalar.

7. Use the integral over the manifold to define the Einstein-Hilbert action, 


\section{Linear Connections}

In the noncommutative case we try as much as possible to mimic the steps of the classical case. Let $\mathcal{A}$ be an arbitrary algebra and $\Omega^{*}(\mathcal{A})$ a differential calculus over it. If $\Omega^{1}(\mathcal{A})$ has a basis $\theta^{\alpha}$ (Stehbein) then one can repeat the first 5 steps above, with a modification of Step 3. We have already mentioned that there are problems with Steps 6. and 7. Since some of the interesting models use differential calculi which do not possess a frame we discuss covariant derivatives in general. We replace Step 3. by

$3^{\prime}$. Define a covariant derivative as a linear map of the form

$$
\Omega^{1}(\mathcal{A}) \stackrel{D}{\rightarrow} \Omega^{1}(\mathcal{A}) \otimes_{\mathcal{A}} \Omega^{1}(\mathcal{A})
$$

This is (13) in a slightly more abstract notation. The subscript on the tensor product means that for an arbitrary element $f$ of $\mathcal{A}$ and elements $\xi$ and $\eta$ of $\Omega^{1}(\mathcal{A})$ one has the identity

$$
\xi f \otimes \eta=\xi \otimes f \eta
$$

The Leibniz rule must be extended since in general

$$
f \xi \neq \xi f \text {. }
$$

\subsection{The Leibniz rules}

Since nothing commutes in general the covariant derivative must satisfy a left Leibniz rule

$$
D(f \xi)=d f \otimes \xi+f D \xi
$$

as well as a right Leibniz rule

$$
D(\xi f)=\sigma(\xi \otimes d f)+(D \xi) f .
$$

The purpose of the map $\sigma$ [34] is to bring the differential to the left while respecting the order of the factors. In the commutative case it is a simple permutation. On the Wess-Zumino calculus over the quantum plane [35] and on the Woronowicz calculus over quantum groups [36] it is given by the $R$-matrix. It is necessarily [35] bilinear,

$$
\sigma(f \xi \otimes \eta)=f \sigma(\xi \otimes \eta), \quad \sigma(\xi \otimes \eta f)=\sigma(\xi \otimes \eta) f
$$

and it must satisfy a compatibility condition [35]. As definition of a linear connection in the noncommutative case we propose the couple $(D, \sigma)$.

\section{Gravity on Fuzzy Spaces}

The definition we propose of gravity on a fuzzy space-time is a simple-minded generalization of the classical case. The only modification is the introduction of the generalized permutation $\sigma$. We first discuss the models and then an example of fuzzy gravity. When there is a generalized Dirac operator $\theta$ it is easy to see that at least one covariant derivative can be defined by the formula

$$
D \xi=-\theta \otimes \xi+\sigma(\xi \otimes \theta)
$$

When $\sigma=-1$ there is a simple relation between the corresponding covariant derivative and the exterior derivative $d$. 


\subsection{The models}

On the first Connes-Lott model there is no covariant derivative with $D^{2} \neq 0$. On the second model there is a 1-parameter family of $\sigma$ and for each $\sigma$ there is a unique covariant derivative, given by (17), which is torsion free and, for one value of the parameter, compatible with a metric [37].

On the matrix models with derivation-based differential calculi there is a natural metric defined by requiring that the frame be orthonormal. There is a natural $\sigma$, defined as a permutation on the frame, with respect to which there is a unique torsion-free, metriccompatible covariant derivative [11, 37]. The covariant derivative (17) has torsion but no curvature.

On the quantum plane with generic $q$ and with the Wess-Zumino calculus the unique $\sigma$ is given in terms of the $R$-matrix. There is, to within a normalization constant $\mu$, a unique covariant derivative. It is without torsion but not metric-compatible. In the limit $q \rightarrow 1$ the covariant derivative yields as curvature a quadratic polynomial in $x$ and $y$ [35]:

$$
R^{i}{ }_{j 12}=4 \mu^{4} A_{j}^{i}, \quad A=\left(\begin{array}{cc}
x y & -x^{2} \\
y^{2} & -x y
\end{array}\right) .
$$

On the extended quantum plane for generic $q$ and for each differential calculus there is a unique torsion-free, metric connection which yields a Gaussian curvature on the plane in the limit $q \rightarrow 1$. In particular the Wess-Zumino calculus is associated to the Gaussian curvature

$$
K=x^{-4}\left(1+y^{4}\right)
$$

This curvature remains as a shadow of the Wess-Zumino calculus.

On the quantum groups $G L_{q}(n)$ for each $\sigma$ the only linear connection for generic $q$ is the one defined by (17). The arbitrariness lies alone in the generalized permutation $\sigma$ for which there exists at least a 2-parameter family [36].

\subsection{Fuzzy gravity}

In defining gravity on a fuzzy space-time we follow [8] closely the example of the extended quantum plane [19]. Consider a $*$-algebra $\mathcal{A}$ with 4 hermitian generators $q^{\mu}$ which satisfy (1) as well as the condition [7]

$$
\left[q^{\lambda}, q^{\mu \nu}\right]=0
$$

We shall suppose that the inverse $q_{\mu \nu}^{-1}$ of $q^{\mu \nu}$ exists: $q_{\lambda \mu}^{-1} q^{\mu \nu}=\delta_{\lambda}^{\nu}$.

Consider the derivations $e_{\lambda}$ of $\mathcal{A}$ given by

$$
e_{\lambda} f=-i \mu_{P}^{2} q_{\lambda \mu}^{-1}\left[q^{\mu}, f\right]
$$

and define a differential as usual by the equation $d f\left(e_{\lambda}\right)=e_{\lambda} f$. The frame is given by

$$
\theta^{\lambda}=d q^{\lambda}
$$

The unique covariant derivative is given by $D \theta^{\lambda}=0$. That is, the algebra is a noncommutative version of Minkowski space, extended by the 'coordinates' $q^{\mu \nu}$.

If one perturbs the relation (20) by

$$
q^{\mu \nu} \mapsto q^{\prime \mu \nu}=q^{\mu \nu}+q_{(1)}^{\mu \nu}
$$

with $\left[q^{\lambda}, q_{(1)}^{\mu \nu}\right]$ in the center of the algebra then there is a rather uninteresting but not completely trivial covariant derivative given by (13) with $\omega^{\lambda}{ }_{\mu \nu}$ given in terms of $q_{\mu \rho}^{-1} q_{\nu \sigma}^{-1}\left[q^{\lambda}, q_{(1)}^{\rho \sigma}\right]$. A preliminary investigation has been made [8] of fuzzy de Sitter space. 


\section{References}

[1] P.A.M. Dirac, Proc. Camb. Phil. Soc. 23 (1926) 412.

[2] A. Connes, Publications of the Inst. des Hautes Etudes Scientifique. 62 (1986) 257.

[3] A. Connes, Noncommutative Geometry, Academic Press, 1994.

[4] H.S. Snyder, Phys. Rev. 71 (1947) 38.

[5] J. Madore, Annals of Physics 219 (1992) 187.

[6] J. Madore, An Introduction to Noncommutative Differential Geometry and its Physical Applications, Cambridge University Press, 1995.

[7] S. Doplicher, K. Fredenhagen and J.E. Roberts, Commun. Math. Phys. 172 (1995) 187.

[8] J. Madore and J. Mourad, Quantum Space-Time and Classical Gravity, Preprint, LPTHE Orsay, (1996) 95/56, gr-qc/9607060.

[9] L. Castellani, Commun. Math. Phys. 171 (1995) 383.

[10] B. de Wit, J. Hoppe and H. Nicolai, Nucl. Phys. B305 (1988) 545.

[11] M. Dubois-Violette, R. Kerner and J. Madore, Phys. Lett. B217 (1989) 485; Class. Quant. Grav. 6 (1989) 1709; J. Math. Phys. 31 (1990) 316.

[12] J. Madore and J. Mourad, Phys. Lett. B359 (1995) 43.

[13] A. Cuntz and D. Quillen, J. Amer. Math. Soc. 8 (1995) 251.

[14] M. Dubois-Violette, J. Madore, T. Masson and J. Mourad, J. Math. Phys. 37 (1996) 4089 .

[15] W. Kalau and M. Walze, Jour. Geom. and Phys. 16 (1995) 327.

[16] T. Ackermann and J. Tolksdorf 1996, A generalized Lichnerowicz formula, the Wodzicki Residue and Gravity, Jour. Geom. and Phys. (to appear).

[17] A. Connes, Gravity coupled with matter and the foundation of noncommutative geometry, IHES Preprint 1996, hep-th/9603053.

[18] A. Chamseddine and A. Connes, A Universal Action Principle, IHES Preprint 1996, hep-th/9606056.

[19] A. Dimakis and J. Madore, J. Math. Phys. 37 (1996) 4647.

[20] S. Deser, Rev. Mod. Phys. 29 (1957) 417.

[21] C.J. Isham, A. Salam and J. Strathdee, Phys. Rev. D3 (1971) 1805.

[22] J. Madore and J. Mourad, Noncommutative Kaluza-Klein Theory, Lecture given at the 5th Hellenic School and Workshops on Elementary Particle Physics, 1995, hepth/9601169. 
[23] A. Connes and J. Lott, Nucl. Phys. Proc. Suppl. B18 (1990) 29.

[24] M. Dubois-Violette, C. R. Acad. Sci. Paris 307 Série I (1988) 403.

[25] J. Madore, J. Mourad and A. Sitarz, On Deformations of Differential Calculi, Mod. Phys. Lett. A (to appear).

[26] J. Madore, Phys. Lett. B263 (1991) 245.

[27] H. Grosse and J. Madore, Phys. Lett. B283 (1992) 218.

[28] H. Grosse and P. Prešnajder, Lett. in Math. Phys. 28 (1993) 239.

[29] H. Grosse, C. Klimčík and P. Prešnajder, Commun. Math. Phys. 178 (1996) 507.

[30] H. Grosse, C. Klimčík and P. Prešnajder, Field Theory on a Supersymmetric Lattice, Preprint, CERN-TH-95-195, hep-th/9507074.

[31] W. Pusz and S.L. Woronowicz, Rep. on Math. Phys. 27 (1989) 231.

[32] J. Wess and B. Zumino, Nucl. Phys. B (Proc. Suppl.) 18B (1990) 302.

[33] S.L. Woronowicz, Publ. RIMS, Kyoto Univ. 23 (1987) 117.

[34] J. Mourad, Class. Quant. Grav. 12 (1995) 965.

[35] M. Dubois-Violette, J. Madore, T. Masson and J. Mourad, Lett. Math. Phys. 35 (1995) 351.

[36] Y. Georgelin, J. Madore, T. Masson and J. Mourad, On the non-commutative Riemannian geometry of $G L_{q}(n)$, Preprint LPTHE Orsay 95/51, q-alg/9507002.

[37] J. Madore, T. Masson and J. Mourad, Class. Quant. Grav. 12 (1995) 1429. 